REVISTA ECONOMÍA

Vol. 69, N. ${ }^{\circ}$ IIO (noviembre), I3-24

\title{
HILANDO LA DESIGUALDAD: GRUPOS ECONÓMICOS Y PARAÍSOS FISCALES EN ECUADOR
}

\author{
JONATHAN BÁEZ
}

Flacso-Ecuador

Recepción de manuscrito: 11 de septiembre de 2017 Aceptación del manuscrito: 23 de octubre de 2017

\begin{abstract}
RESUMEN El siguiente artículo tiene como objetivo describir empíricamente la relación que los grupos económicos tienen con los paraísos fiscales en Ecuador. ${ }^{1}$ La evidencia muestra que los grupos económicos del quintil más alto de ingresos son los que poseen el mayor número de integrantes domiciliados en paraísos fiscales. Además, se observa que por cada integrante que se encuentre domiciliado en un paraíso fiscal, un grupo económico dejaría de pagar en promedio USD 1,96 millones de impuestos. En este sentido, los grupos económicos de mayores ingresos utilizan a los paraísos fiscales como una herramienta para de reforzar la desigualdad.
\end{abstract}

PALABRAS ClAVE Evasión fiscal, equidad, justicia, desigualdad, renta personal.

ABSTRACT The following article aims to empirically describe the relationship between business conglomerates and tax havens in Ecuador. The evidence shows that the highest quintile income conglomerates are those with the most members residing in tax havens. In addition, for each member in a tax haven, a conglomerate would save an average of USD 1.96 million in taxes, with higher-income economic groups using tax havens as an tool to reinforces inequality. KEYWORDS Tax evasion, equity, justice, inequality, personal income

Jel CODeS H26, D63, D31.

\section{INTRODUCCIÓN}

La investigación de los Panama papers realizada por el Consorcio Internacional de Periodistas de Investigación y divulgada en abril de 2016, situó la problemática de los paraísos fiscales en el centro del debate sobre justicia fiscal. La mencionada investigación develó que la firma Mossack Fonseca — con sede en Panamá - creó cerca de 240 mil sociedades que permitía «ocultar el origen ilícito del dinero, contribuir a la evasión fiscal, la fuga de capitales y para el lavado del dinero». (Grondona, 2016). Sin embargo, es importante resaltar que si en la lista de los Panama papers aparecen políticos y celebridades, son pocas multinacionales las que figuran en dicha lista (Grondona, 2017).

El efecto del escándalo de los Panama papers tuvo repercusiones en varios países, pero sobre todo en Ecuador, ${ }^{2}$ ya que el 6 de julio de 2017 se aprobó el proyecto de Ley sobre Paraísos 
Fiscales por la Asamblea Nacional. Este suceso ocurre en el marco de la victoria del sí en la consulta popular del 19 de febrero del mismo año en el que se establece la prohibición del uso de paraísos fiscales a servidores públicos y dignatarios. ${ }^{3}$ Si bien este es un importante avance hacia la justicia fiscal en tanto «los paraísos fiscales se han convertido en una de las principales causas, sin precedentes, de la desigualdad mundial al permitir que individuos ultra ricos y corporaciones evadan los impuestos que deben pagar» (Ministerio de Relaciones Exteriores y Movilidad Humana, 2017, p. 4) al igual que ocurrió con los Panama papers, la problemática de la desigualdad económica en sí misma parece no abordarse. En este sentido, es pertinente preguntarse si en el caso ecuatoriano: ¿Existe evidencia que sustente la relación entre grupos de poder, desigualdad y paraísos fiscales? ¿Cómo los paraísos fiscales configuran y profundizan la desigualdad económica? Es decir, ¿cuál es la relación entre grupos económicos y paraísos fiscales?

El presente artículo intenta responder las preguntas planteadas. En un primer momento se realiza un repaso sobre los estudios que muestran, por un lado, la concentración económica de los grupos económicos y, por otro, su relación con el pago de impuestos. Después se describe la relación que los grupos económicos ecuatorianos poseen con los paraísos fiscales y cómo estos últimos funcionan como mecanismos de disminución en el pago de impuestos.

\section{CONCENTRACIÓN Y GRUPOS ECONÓMICOS EN ECUADOR}

La problemática de la concentración retornó al centro del debate mundial a partir del estudio realizado por Piketty (2014), en el que demuestra que la economía tiene una tendencia hacia la concentración de ingresos, pero sobre todo de riqueza. En América Latina pueden destacarse los estudios realizados por Solimano (2009 y 2015). En ambos autores, la presencia de las élites económicas propicia y profundiza la concentración y, por tanto, la desigualdad. De esta manera, son las elites económicas - en especial las empresariales - las que son objeto de estudio y análisis. Así «las élites empresariales en América Latina han organizado sus dominios en forma de grupos económicos, que se definen como conjuntos de empresas relacionadas entre sí por estar controladas por una entidad en común». (Cárdenas 2016, p. 3). De esta forma, en América Latina los grupos económicos se convierten en el eje de análisis en estudios sobre concentración y el caso de Ecuador no es la excepción.

La temática de los grupos económicos ha sido abordada en el Ecuador desde una perspectiva que se relaciona con los altos niveles de concentración económica que estos actores tienen. En los años 70, el estudio realizado por Navarro (1976) inaugura este tipo de estudios en el que reconoce a los grupos económicos como núcleos familiares que «controlan un número importante de empresas, las que se localizan en varias provincias y en varias ramas de actividad». (Navarro, 1976, p. 57). ${ }^{4}$ No obstante, este estudio focaliza la concentración que los grupos familiares tienen sobre las empresas y no la que éstas tienen sobre el conjunto de la economía. En esa misma línea de investigación, el Centro de Estudios y de Difusión Social (CEDIS) (1986) identifica a los grupos económicos más importantes del país. Sin embargo, también indica los niveles de concentración y centralización de capital ${ }^{5}$ de estos grupos en distintas ramas de la economía ecuatoriana, mostrando la tendencia monopólica de estos grupos en activos 
Tabla 1. Estructura de ingresos e impuestos por quintiles de grupos económicos

\begin{tabular}{|c|c|c|c|c|c|c|c|c|}
\hline & $\mathrm{N} .^{\circ}$ & $\begin{array}{l}\text { Integrantes } \\
\text { domiciliados } \\
\text { en paraísos } \\
\text { fiscales }\end{array}$ & $\begin{array}{c}\text { Integrantes y } \\
\text { empresas } \\
\text { offshore } \\
\text { Panama Papers }\end{array}$ & $\begin{array}{c}\text { Total } \\
\text { ingresos } \\
\text { (millones USD) }\end{array}$ & $\begin{array}{l}\text { Impuesto a la } \\
\text { renta causado } \\
\text { (millones USD) }\end{array}$ & $\begin{array}{c}\text { Total } \\
\text { recaudado en } \\
\text { impuestos } \\
\text { (millones USD) }\end{array}$ & $\begin{array}{c}\text { Relación } \\
\text { impuesto a la } \\
\text { renta causado/ } \\
\text { total ingresos }\end{array}$ & $\begin{array}{l}\text { Relación total } \\
\text { recaudado en } \\
\text { / impuestos / } \\
\text { total ingresos }\end{array}$ \\
\hline Quintil 1 & 40 & 33 & 46 & 1148 & 33 & 310 & $2,85 \%$ & $26,97 \%$ \\
\hline Quintil 1 & 40 & 35 & 67 & 3330 & 88 & 300 & $2,64 \%$ & $9,01 \%$ \\
\hline Quintil 1 & 40 & 52 & 29 & 6631 & 177 & 731 & $2,67 \%$ & $11,02 \%$ \\
\hline Quintil 1 & 40 & 115 & 60 & 12258 & 256 & 1325 & $2,09 \%$ & $10,81 \%$ \\
\hline Quintil 1 & 40 & 135 & 147 & 34109 & 775 & 3596 & $2,27 \%$ & $10,54 \%$ \\
\hline Total & 200 & 370 & 349 & 57476 & 1329 & 6262 & $2,31 \%$ & $10,89 \%$ \\
\hline
\end{tabular}

Fuente: SRI. Elaboración: propia.

productivos - tierra, capital, mano de obra, etc.- y en mercados específicos. Finalmente, el estudio de Fierro (1991), en forma paralela pero distinta a CEDIS, se centra en identificar específicamente los sectores de la economía en donde los grupos económicos se han consolidado y generan tendencias oligopólicas o monopólicas en las ventas. En este sentido se muestra que un reducido número de empresas concentran un nivel importante del nivel de ventas y que dichas empresas, en la mayoría de los casos, pertenece a algún grupo económico. Por consiguiente, se menciona que «apenas el 3 por ciento de las empresas controló el 53 por ciento de las ventas, en 21 ramas seleccionadas, las compañías monopólicas constituyen el 16 por ciento del total de las empresas y concentran el 62\% de las ventas». (Fierro, 1991, p. 141).

Los estudios que se realizan en los años siguientes continúan la misma línea planteada por Fierro. En otras palabras, se comparan los niveles de ventas de las empresas que pertenecen a los grupos económicos en distintas ramas y se demuestra que existe concentración en los mencionados sectores. Así, se pueden indicar los estudios realizados a nivel general -la totalidad de sectores - por Unda y Bethania (2010), Delgado (2010), EKos (2012), Cañas (2014, 2015) y Pástor (2015). Por otra parte, el estudio de los grupos económicos en sectores específicos ha sido el predominante en la actualidad ecuatoriana, entre los que destacan los trabajos de Iturralde (2014) en el sector salud, Montalvo (2014) en el sector automotriz y Báez (2017) en el sector agropecuario. ${ }^{6}$

\section{GRUPOS ECONÓMICOS ECUATORIANOS Y PARAÍSOS FISCALES}

El uso de los paraísos fiscales utilizado por los grupos de poder ha sido abordado durante los últimos años: Zucman, 2015; O’Donovan, Wagner y Zeume, 2016. De esa manera, se estima que «por lo menos USD 21 billones de la riqueza financiera privada se refugia en este tipo de jurisdicciones» (Henry, 2012; citado en Ordóñez, 2017, p. 5). En otro estudio se indica que «cada año los países en desarrollo pierden al menos USD 100.000 millones por abusos fiscales de grandes transnacionales, tanto por la evasión y elusión fiscal que se efectiviza a través de los paraísos fiscales» (oxfAM, 2015; citado en Ordóñez, 2017 p. 6). Desde esa misma perspectiva Fuest y Riedel (2010) demuestran que: 
[...] Las empresas multinacionales en general y las que tienen una conexión de paraíso fiscal pagan impuestos más bajos sobre sus beneficios [...] en comparación con las empresas nacionales [...] Los resultados sugieren que las empresas multinacionales pagan 1 punto porcentual menos impuestos sobre sus ganancias antes de impuestos que las corporaciones nacionales, mientras que las empresas multinacionales con una conexión con paraísos fiscales pagan 4,4 puntos porcentuales menos impuestos sobre sus ganancias. (p. 25)

En el caso del Ecuador, los estudios mencionados en la sección anterior muestran cómo la economía está concentrada por los grupos económicos. No obstante, las investigaciones que se realizan desde 2010 empiezan a cuestionar no solo los altos niveles de concentración, sino también discuten el bajo nivel de impuestos que los grupos económicos tributan anualmente. Así, por ejemplo, se menciona que si bien «estos conglomerados aportaron con el 26,5\% de la recaudación de impuesto a la renta (IR) total en 2010; su carga fiscal (impuestos/ingresos) es tan solo de 2,04\%.» (EKOS, 2012, p. 36).

En un análisis similar se determinó que «en el país existen 59 grupos económicos con sociedades domiciliadas en paraísos fiscales, cuyos ingresos para el 2014 fueron más de 28 mil millones de dólares, pero su impuesto a la renta fue de 609 millones, es decir, un 2,15 \% de contribución del impuesto a la renta total de ese año». (Iturralde, 2016, p. 2). De forma paralela, en el análisis de la relación entre grupos económicos vinculados a la agroindustria y paraísos fiscales se indica que éstas «registraron ingresos superiores a los USD 4887 millones en el 2014, mientras que el nivel de impuesto a la renta alcanzó los USD 59 millones, es decir, apenas el 1,2\% de sus ingresos se destinó al pago de este impuesto». (Báez e Iturralde, 2016, p. 1).

Los últimos dos análisis no solo puntualizan el bajo nivel de impuestos pagados por los grupos económicos, sino que sugieren a este fenómeno como un efecto del uso de paraísos fiscales por parte de estos grupos. De esta manera se observa que, del total de integrantes extranjeros que conforman los grupos económicos en el año 2013, alrededor del 54\% están domiciliados en paraísos fiscales (Cañas, 2015, p. 103). Por consiguiente, se observa la importancia de este mecanismo de elusión y evasión fiscal en los grupos económicos del Ecuador.

En los análisis presentados existe un vínculo ambiguo entre grupos económicos y paraísos fiscales. Si bien se muestra las posibles relaciones y efectos del uso de paraísos fiscales como un mecanismo de elusión y evasión de impuestos, ${ }^{7}$ no se indica el impacto cuantitativo que dichos paraísos fiscales causan en términos tributarios.

Por otro lado, tampoco se muestra la heterogeneidad de los grupos económicos y si el uso de paraísos fiscales es similar en todos los grupos o si existen diferencias entre ellos. De igual forma ocurre con los paraísos fiscales, no se presentan las distinciones entre ellos. Este artículo tiene como objetivo llenar estos vacíos a través de la evidencia empírica presentada a continuación.

\section{METODOLOGÍA}

La información que se utiliza en este artículo proviene de la base de datos Grupos Económicos. Conformación y Comportamiento Tributario del año 2015 realizada por el Servicio de Rentas Internas (SRI). Esta base contiene a 200 grupos económicos, identificados a partir de la definición 
Tabla 2. Paraísos fiscales utilizados por los grupos económicos ecuatorianos

\begin{tabular}{lccc}
\hline & Número & Porcentaje & $\begin{array}{c}\text { Índice de } \\
\text { secreto financiero }\end{array}$ \\
\hline Panamá & 212 & 57 & 415,7 \\
Islas Vírgenes (UK) & 50 & 14 & 307,7 \\
Bahamas & 30 & 8 & 273,1 \\
Luxemburgo & 22 & 6 & 817,0 \\
Bermuda & 19 & 5 & 217,7 \\
Islas Caimán & 12 & 3 & 1013,2 \\
Malta & 6 & 2 & 67,8 \\
Curazao & 5 & 1 & 202,4 \\
Liechtenstein & 4 & 1 & 79,7 \\
San Vicente y Las Granadinas & 4 & 1 & 298,3 \\
Barbados & 3 & 1 & 92,5 \\
Belice & 1 & 0 & 213,9 \\
Chipre & 1 & 0 & 118,2 \\
Islas Vírgenes (EE. Uu.) & 1 & 0 & \\
\hline
\end{tabular}

Fuente: Tax Justice Network, 2015). Elaboración: propia.

del SRI de grupos económicos que, de acuerdo con el artículo 5 del Reglamento para la aplicación de la Ley de Régimen Tributario Interno, para fines tributarios se define como grupo económico al conjunto de partes, conformado por personas naturales y sociedades, tanto nacionales como extranjeras, donde una o varias de ellas posean directa o indirectamente $40 \%$ o más de la participación accionaria en otras sociedades. (Servicio de Rentas Internas, 2017). Las principales variables - del año 2015- que serán utilizadas son: i) total de ingresos; ii) impuesto a la renta causado; ${ }^{8}$ iii) total recaudado por todos los impuestos; ${ }^{9}$ iv) integrantes — nacionales, extranjeros-. En el análisis se clasificó a los grupos económicos por quintiles de ingresos, donde el quintil 1 se refiere a los grupos económicos con los menores ingresos y el quintil 5 a los grupos económicos de mayores ingresos. En la consideración de paraísos fiscales se adoptó la definición del SRI (2016) y se reconocen a 86 jurisdicciones como paraísos fiscales en el año 2016, que son espacios geográficos con las siguientes características:

[...] Baja o nula imposición, normas de secreto para evitar el intercambio de información, falta de transparencia en disposiciones legislativas, jurídicas o administrativas y que su legislación requiera, para acogerse a los beneficios, que no exista presencia económica en su territorio o no se requiera sustancia económica para los mismos fines. (p. 4)

El artículo también se refiere a las sociedades registradas en los Panama Papers para lo cual se retomó a los integrantes ubicados por el SRI en la base antes mencionada y que corresponde a la investigación realizada por el Consorcio Internacional de Periodistas de Investigación. De igual manera se incluye en el análisis del Índice de secreto financiero del año 2015 - creado por la coalición Tax Justice Network- ${ }^{10}$ para mostrar las diferencias entre paraísos fiscales y los que más son 
utilizados por los grupos económicos. ${ }^{11}$ Entre mayor sea este índice mayor es la opacidad fiscal de las jurisdicciones, por lo que es más probable que la evasión y elusión tenga lugar. Finalmente se realiza una regresión lineal de corte transversal que muestra la relación entre el nivel de impuestos recaudados y la presencia de paraísos fiscales en los grupos económicos, que permitirá tener evidencia de si estas jurisdicciones opacas tienen incidencia en el pago de impuestos.

\section{RESULTADOS}

El análisis de los grupos económicos del año 2015 (ver Tabla 1) muestra que existen diferencias importantes entre ellos. De esta forma, se observa que el ingreso total de los grupos económicos que pertenecen al quintil 5 es de USD 34.109 millones y el del quintil 1 asciende a USD 1148. Es decir, existe una brecha del $97 \%$ en los ingresos totales entre el quintil con mayores ingresos y el de menores ingresos. Este hecho indica que existe una heterogeneidad importante entre los grupos económicos y que algunos poseen ingresos extraordinarios. En este sentido, si se comparan entre estos mismos quintiles los montos por impuesto a la renta causado y el total recaudado por impuestos, las brechas son del $96 \%$ y $91 \%$ respectivamente.

Dichos datos indicarían que existe cierta progresividad — mientras más elevado sean los ingresos, superiores son los impuestos - en el caso de los grupos económicos. Sin embargo, al analizar la relación entre el impuesto a la renta causado y el total de ingresos de cada quintil, existe una tendencia declinante y los quintiles de grupos económicos con mayores ingresos son los que menos impuestos pagan en proporción con sus ingresos. En los quintiles 4 y 5 se genera un impuesto a la renta que corresponde al 2,09\% y 2,27\% respectivamente; en los quintiles 1 y 2 esta proporción es del 2,85\% y 2,64\%. Las diferencias, si bien son mínimas, son significativas.

La misma tendencia se observa al analizar el total recaudado de impuestos en relación a los ingresos totales, así los grupos económicos del quintil 5 son los que menos pagan proporcionalmente: 10,54\%, en comparación con los del quintil 1: 26,97\%. Entre ambos tipos de quintiles existe una diferencia de 16,43 puntos porcentuales en la mencionada relación. No obstante, ésta no es la única diferencia entre los quintiles de mayores y menores ingresos, también se muestran diferencias con el número de integrantes ubicados en paraísos fiscales y sociedades offshore registradas en los Panama Papers.

Existe evidencia de que los grupos económicos ubicados en el quintil 5 son los que poseen más integrantes domiciliados en paraísos fiscales, con un total de 135 sociedades. Por otro lado, los grupos económicos del quintil 1 poseen apenas 33 integrantes domiciliados en los paraísos fiscales. En otras palabras, los grupos económicos del quintil con mayores ingresos concentran el $36 \%$ del total de integrantes ubicados en paraísos fiscales y los del quintil con menores ingresos absorben apenas al 9\% del total. Una tendencia similar se observa en los integrantes que se encuentran registrados en los Panama Papers. Sin embargo, aquí la diferencia es superior y los grupos económicos del quintil 5 concentran el $42 \%$ del total mientras que los del quintil 1 poseen solo el $13 \%$.

En este punto, es necesario señalar que el uso de los paraísos fiscales también está concentrado. El principal paraíso fiscal que integra los grupos económicos es Panamá y corresponde al 57\% del total de integrantes domiciliados en esta jurisdicción (ver Tabla 2). Es seguido por 
Tabla 3. Determinantes del total del impuesto recaudado (modelo de regresión lineal)

\begin{tabular}{lrc}
\hline Variables & Coeficientes & Std. Err. \\
\hline Total de ingresos & 0,1143848 & $0,01100^{*}$ \\
Número de Sociedades Nacionales & 300853 & $183651^{* *}$ \\
Integrantes domiciliados en paraísos fiscales & -1962150 & $106687^{* *}$ \\
Impuesto a la renta causado & 4437513 & $1686959^{*}$ \\
Integrantes offshore registradas en los Panama Papers & -1493441 & $853515^{* *}$ \\
Constante & $1,33 e+07$ & $705051^{* *}$ \\
R-cuadrada & & 0,4387 \\
R-cuadrada ajustada & & 0,4240 \\
N & & 200
\end{tabular}

Nota: ${ }^{*} \mathrm{P}<0,01 ;{ }^{* *} \mathrm{P}<0,1$. Fuente: SRI. Elaboración: propia

las Islas Vírgenes y Bahamas con el 14\% y $8 \%$ respectivamente. Si se considera el nivel de opacidad de estos tres paraísos fiscales, según el Índice de secreto financiero, el más utilizado Panamá- es el que posee el índice de mayor nivel, 415,7, en comparación con los otros dos. Por consiguiente, se muestra que el paraíso fiscal más utilizado es el que ofrece mayores ventajas para el ocultamiento del dinero. Sin embargo, existen otros paraísos fiscales que poseen un índice más alto pero que conforma a los grupos económicos en un nivel menor. Este es el caso de Islas Caimán con un índice de 1013,2.

En resumen, si los grupos económicos del quintil de mayores ingresos son los que más integrantes en paraísos fiscales poseen, es en aquellos paraísos que ofrecen mayor opacidad los que más se utilizan. De esta manera se configura un proceso en el que la evasión y elusión fiscal es más probable en los grupos económicos y, de mayor forma, en los grupos económicos de los quintiles con los más altos ingresos.

Los datos presentados hasta el momento muestran cómo los grupos económicos ubicados en el quintil de mayores ingresos son los que menores niveles de impuestos - renta causada y sobre todo del total recaudado- pagan en relación con sus niveles de ingresos. Además, estos mismos grupos son los que poseen más integrantes domiciliados en paraísos fiscales de mayor opacidad - y todavía más en los integrantes vinculados a la lista de Panama Papers. De esta forma se demuestra que los grupos económicos del quintil más alto de ingresos son los que más se relacionan con paraísos fiscales y un bajo nivel de tributación —al compararlos con sus ingresos - en el país. A pesar que de este análisis se observa una posible relación, es necesario comprobar que existe tal relación empíricamente. Es decir, determinar los componentes del nivel de impuestos recaudados y si la presencia de integrantes domiciliados en paraísos fiscales tiene alguna incidencia en el nivel de recaudación. Por consiguiente, se construye una regresión en la que se forma la siguiente ecuación:

$$
T_{R}=\beta_{0}+\beta_{1} \operatorname{Ing}+\beta_{2} S n+\beta_{3} I P f+\beta_{4} I R c+\beta_{5} I O f+U_{i}
$$

Donde:

$T_{R}=$ total impuesto recaudado;

Ing = total de ingresos; 
$S n$ = número de Sociedades Nacionales;

$I P f=$ integrantes domiciliados en paraísos fiscales;

$I R c=$ impuesto a la renta causado;

IOf $=$ integrantes offshore registradas en los Panama Papers

$U_{i}=$ error de estimación

A pesar de tratarse de una regresión lineal simple y de sus limitaciones, se demuestra la presencia de la relación entre grupos económicos, paraísos fiscales y un bajo nivel de impuestos. En este sentido, los resultados de la regresión indican (ver Tabla 3) que las variables son estadísticamente significativas tanto individualmente como en conjunto. Las variables que poseen signo positivo son: i) el total de ingresos; ii) el número de sociedades nacionales; y iii) el nivel de impuesto a la renta causado. Por otra parte, las variables con signo negativo son: i) integrantes domiciliados en paraísos fiscales y ii) integrantes offshore registrados en los Panama Papers. Estos datos demuestran que existe una relación inversa entre el total recaudado y la presencia de paraísos fiscales e integrantes offshore en los grupos económicos ecuatorianos.

El efecto marginal indica que - ceteris paribus - ${ }^{12}$ si en un grupo económico el número de integrantes domiciliado en un paraíso fiscal aumentan en uno, en promedio el nivel de recaudación total de impuestos disminuye en cerca de USD 1,96 millones. Por otro lado, si los integrantes que se encuentran en los registros de Panama Papers aumentan en uno, el nivel promedio de recaudación total de impuestos disminuye en promedio cerca de USD 1,49 millones.

En un sentido contrario, si el número de sociedades domiciliadas en territorio nacional ecuatoriano- aumenta en uno, un grupo económico pagaría por impuestos — en promedioalrededor de USD 300 mil adicionales. Es decir, existe un cierto tipo de «castigo» a los grupos económicos que no poseen integrantes domiciliados en paraísos fiscales. Lo que sucede en realidad es que los grupos económicos que poseen este tipo de integrantes tienen una alta probabilidad de poder realizar operaciones financieras que sirven de escudos fiscales para disminuir el pago de impuestos, por el hecho de que se componen de este tipo de integrantes. ${ }^{13}$

En consecuencia, la presencia de paraísos fiscales incide negativamente en la recaudación tributaria. Por consiguiente, se demuestra que la relación entre paraísos fiscales y grupos económicos determina que el total de impuestos recaudados disminuya considerablemente. Si se considera que los grupos económicos de mayores ingresos son los que más integrantes domiciliados en paraísos fiscales poseen, son estos grupos los que están hilando la desigualdad, utilizando a estos paraísos como un instrumento que les permite no pagar impuestos y que actúan, por tanto, como un mecanismo para aumentar la desigualdad.

\section{CONCLUSIONES}

Los datos señalados indican que los grupos económicos concentran la economía del Ecuador. A esto debe agregarse que aquellos que pertenecen al quintil de mayores ingresos son los que más utilizan a los paraísos fiscales como un instrumento que les permite el no pago de impuestos. Así, se demuestra que existe una relación empírica entre los paraísos fiscales y el uso que los grupos económicos hacen de este instrumento. Por otra parte, dada la estructura regresiva de América Latina y del Ecuador en materia tributaria, los contramecanismos para disminuir 
la desigualdad se observan todavía muy limitados. En este sentido es pertinente preguntarse si es suficiente la aspiración de que no se permita el uso de paraísos fiscales a servidores públicos o es una medida más efectiva que se condicione a los grupos económicos de mayores ingresos el uso de paraísos fiscales, como medida de disminución de la desigualdad.

\section{NOTAS}

1 Deseo agradecer los comentarios del economista Guido Duque Phd (c) en los distintos borradores del presente artículo, así como a Carlos Bedoya coordinador de la Red Latinoamericana sobre Deuda, Desarrollo y Derechos (LATINDADD) y a Pablo Iturralde, del Centro de Derechos Económicos y Sociales (CDEs). 2 En Ecuador se indica - según datos de dicha lista- que «al menos 40 empresas ecuatorianas presuntamente realizaron transferencias de pago a la firma legal panameña Mossack Fonseca». (Telesur, 2016). 3 La pregunta de la consulta fue: «¿Está usted de acuerdo en que, para desempeñar una dignidad de elección popular o para ser servidor público, se establezca como prohibición tener bienes o capitales, de cualquier naturaleza, en paraísos fiscales?» (El Universo, 2017). Un 55,12\% de las personas habilitadas para votar estuvo a favor del sí frente a un 44,88\% que no lo estuvo. (El Universo, 2017; Revista Vistazo, 2017; El Comercio, 2017). 4 En ese sentido se indica que «una sola familia controla hasta 13 empresas» (Navarro, 1976, p. 59).

5 La concentración de capital es el proceso a través del cual ciertas empresas logran acumular la mayor parte de la producción en un periodo determinado debido a una articulación entre sus economías de escala y funcionamiento del sistema en conjunto - monopólico u oligopólico- - . Por otra parte, la centralización de capital se refiere al proceso en que los capitales autónomos en constante competencia logran absorber o destruir a otros fortaleciéndose, tendencia lógica del sistema de mercado capitalista. Para ver más sobre la diferencia entre estos dos procesos se recomienda ver Aglietta (1979).

6 Incluso en el conjunto de la contratación pública se observa la existencia de concentración por parte de empresas que pertenecen a grupos económicos. Como lo demuestra Báez (2015) al señalar que estas empresas se encuentran en el ranking con los mayores ingresos en la contratación pública.

7 «La diferencia principal entre elusión y evasión radica en que, en la elusión el contribuyente busca impedir el nacimiento de la obligación tributaria, a través de medios lícitos aunque infrecuentes ubicándose en una zona a donde la ley tributaria no llega; en cambio, en la evasión nace la obligación tributaria, pero el contribuyente mediante una conducta fraudulenta impide que la Administración Tributaria la conozca, o busca reducir su verdadero monto». (Santiana, 2013, p. 27).

8 Corresponde al mayor valor entre el impuesto a la renta causado declarado y el impuesto mínimo pagado a través del anticipo de impuesto a la renta, más el impuesto a la renta único para la actividad productiva de banano, según corresponda.

9 Se refiere al total recaudado, a través de los formularios de declaraciones, tanto como agentes de percepción y retención. No contempla IVA e ICE importaciones.

10 La Tax Justice Network «es una entidad internacional enfocada en la investigación del rol de los impuestos y el impacto negativo de la evasión, elusión y los paraísos fiscales. El índice de secreto financiero califica a 92 jurisdicciones a nivel mundial, y se calcula en base a dos aspectos: 1) la parte cualitativa, que analiza las leyes, regulaciones y tratados de cada jurisdicción para evaluar el nivel de opacidad y asignar el puntaje a cada país, entre mayor el puntaje, más opaca la jurisdicción; 2) la parte cuantitativa, que asigna una ponderación a cada país de acuerdo a su participación en el mercado global de servicios financieros offshore». (Ordóñez, 2017, p. 3).

11 Una descripción específica de este índice y de su metodología puede observarse en Meinzer (2010).

12 Ver Gujarati y Porter (2009).

13 Las grandes empresas en Ecuador poseen índices de rentabilidad neta menores a los de microempresas. Esto ocurre porque las primeras disminuyen su utilidad para que el pago de impuesto sea menor y, también, para disminuir el pago de utilidades a trabajadores. Lo que influye en el índice. Ver Báez (2016). 


\section{REFERENCIAS}

Aglietta, M. (1979). Regulación y crisis del capitalismo. La experiencia de los Estados Unidos. México DF, México: Siglo xxi.

Báez, J. (2016). Género y etnia en la política fiscal. Centro de Derechos Económicos y Sociales. Recuperado de http://prueba.justiciafiscal.org/2016/o6/o7/cdes-genero-y-etnia-en-la-politica-fiscal/

Báez, J. (2016, agosto 14). ¿Para quién el Estado? Estudio de la concentración en compras públicas. Centro de Derechos Económicos y Sociales. Recuperado de http://cdes.org.ec/web/nueva-investigaci on-para-quien-el-estado-estudio-de-la-concentracion-en-compras-publicas/

Báez, J. (2016, marzo 22). Paro parcial o parcialidad del ingreso. La línea de fuego. Recuperado de https:// lalineadefuego.info/2016/o3/22/paro-parcial-o-parcialidad-del-ingreso-por-jonathan-baez1/

Báez, J. (2017). Acumulación en la revolución ciudadana: Grupos económicos y concentración de mercado en el sector agropecuario. Quito, Ecuador: (s. d.).

Báez, J. e Iturralde, P. (2016, mayo 10). Agroindustria en paraísos fiscales de los Panama papers. La línea de fuego. Recuperado de https://lalineadefuego.info/2016/05/10/agroindustria -en-parai sos-fiscales-de-los-panama-papers-por-jonathan-baez-y-pablo-iturraldei/

Cañas, R. (2014). El proceso de internacionalización de los grupos económicos del Ecuador: una perspectiva histórico-económica. (Tesis de maestría). Pontificia Universidad Católica del Ecuador, Quito, Ecuador.

Cañas, R. (noviembre, 2015). Caracterización de los grupos económicos en el Ecuador. Investigación económica, (8), 76-114.

Cárdenas, J. (2016). Enredando a las elites empresariales en América Latina: análisis de redes de interlocking directorates y propiedad en México, Chile, Perú y Brasil. América Latina hoy, 73, 15-44.

Centro de Estudios y Difusión Social (CEDIS) (1986). Los grupos monopolicos en el Ecuador: un ensayo de difusión popular. Quito, Ecuador: CEDIS.

Con 107 votos a favor Asamblea aprueba Ley de Paraísos Fiscales (6 de julio de 2017). El Universo. Recuperado de https://www.eluniverso.com/noticias/2017/o7/o6/nota/6267091/107 -votos-favor -se-aprobo-ley-paraisos-fiscales-asamblea-nacional

Cuarenta (40) empresas ecuatorianas involucradas en los Panama Papers. (2016, mayo 9). Telesur. Recuperado de https://www.telesurtv.net/news/40-empresas-ecuatorianas-involucradas-enlos-Panama-Papers--20160509-0032.html

Delgado, A. (2010). La concentración de capitales en Ecuador y sus efectos, período 2002-2008. (Tesis de maestría). Pontificia Universidad Católica del Ecuador, Quito, Ecuador.

EKOS (2012, marzo 12). Los colosos de la economía nacional. EKOs Negocios, (215), 28-70.

El sí gana en la consulta de los paraísos fiscales pedida por Alianza País. (21 de febrero de 2017). El Comercio, (s. d.).

Este domingo 19 también hay consulta popular sobre paraísos fiscales. (17 de febrero de 2017). El Universo. Recuperado de https://www.eluniverso.com/noticias/2017/02/17/nota/6052039/ este-domingo-19-tambien-hay-consulta-popular-sobre-paraisos

Fierro, L. (1991). Los grupos financieros en el Ecuador. Quito, Ecuador: Centro de Educación Popular.

Financial Secrecy Index 2015. (2015, noviembre 2). Tax Justice Network. Recuperado de http://www.taxjustice.net/wp-content/uploads/2013/o4/FsI-2015-Presser.pdf.

Fuest, C. and Riedel, N. (2010). Tax evasion and tax avoidance in developing countries: the role of international profit shifting. Oxford, UK: Oxford University Centre for Business Taxation. 
Gana el «sí» en consulta sobre el «pacto ético», dice CNE. (2017, marzo 1). Revista Vistazo. Recuperado de http://www.vistazo.com/seccion/elecciones-2017-noticias-elecciones/noticias-elecciones/ gana-el-si-en-consulta-sobre-el

Grondona, V. (2016, abril 6). Los Panama Papers y el rol de las guaridas fiscales. Red de Justicia Fiscal de América Latina y el Caribe Red de Justicia Fiscal de América Latina y el Caribe. Recuperado de http://www.justiciafiscal.org/2016/o4/los-panama-papers-y-el-rol-de-las-guaridas-fiscales/

Grondona, V. (2017, enero 13). Panamá Papers tensó el debate europeo. Fundación ses. Recuperado de https://www.sesamericalatina.com/single-post/2017/o1/13/Panam\%C3\%1-Papers-tens\% $\mathrm{C}_{3} \% \mathrm{~B}_{3}$-el-debate-europeo

Gujarati, D. y Porter, D. (2009). Econometría. México DF, México.: McGraw-Hill.

Iturralde, P. (2014). El negocio invisible de la salud: Análisis de la acumulación de capital en el sistema de salud del Ecuador. Quito, Ecuador: Arcoiris-Producción Gráfica.

Iturralde, P. (2016, abril 6). A propósito de los Panama Papers: 59 grupos económicos del país fueron vinculados con paraísos fiscales. Centro de Derechos Económicos y Sociales. Recuperado de http:// cdes.org.ec/web/59-grupos-economicos-mantienen-vinculos-con-paraisos-fiscales/

Meinzer, M. (2010). Contribución para el Observatorio del Instituto AfIP. Tax Justice Network. Recuperado de http://www.taxjustice.net/cms/upload/Meinzer2010_Observatorio_11.5.2010.pdf

Ministerio de Relaciones Exteriores y Movilidad Humana. (2017). Ecuador líder mundial contra los paraísos fiscales. (s. d.).

Montalvo, J. (2014). Análisis sobre la concentración de poder de los grupos económicos del sector automotriz (ensamblaje y comercialización) en el Ecuador. (Tesis de maestría). Pontifice Universidad Católica del Ecuador, Quito, Ecuador.

Navarro, G. (1976). La concentración de capitales en Ecuador. Quito, Ecuador: Ediciones Solitierra.

O'Donovan, J., Wagner, H. F. and Zeume, S. (2016, abril 28). The value of offshore secrets. Evidence from the Panama Papers. SSRN. Recuperado de https://papers.ssrn.com/sol3/papers.cfm?abstract _id $=2771095$

Ordóñez, M. (2017). Lucha contra paraísos fiscales: una mirada crítica. Quito, Ecuador: Centro de Estudios Fiscales.

Pástor, C. (2015). Los grupos económicos en el Ecuador. (Tesis de maestría). Universidad Andina Simón Bolívar, Quito, Ecuador.

Piketty, T. (2014). Capital in the twenty-first century. Cambridge, usA; London, UK: The Belknap Press of Harvard University.

Santiana, A. V. (2013). Medidas tendientes a combatir la elusión y la evasión fiscal de impuesto a la renta de las sociedades en el Ecuador. (Tesis de maestría). Pontifice Universidad Católica del Ecuador, Quito, Ecuador.

Servicio de Rentas Internas (SRI) (2016). Paraísos y regímenes fiscales preferentes. Resolución. Quito, Ecuador. (s. d.).

Solimano, A. (2009, diciembre 20). Concentración Económica, heterogeneidad productiva, políticas públicas y contrato social en Chile. сіgLoв. Santiago de Chile, Chile. Recuperado de http://www. ciglob.org/wp-content/uploads/2016/o9/WP_13.pdf

Solimano, A. (2015). Elites económicas, crisis y el capitalismo del siglo XXI. La alternativa de la democracia económica. Santiago de Chile, Chile: FCE-Chile s. A. 
Unda, M. y Bethania, E. (2010). Burguesía ciudadana, nueva hegemonía, nueva alianza de clases. Revista $R$, (s. d.), 19-31.

Zucman, G. (2015). La riqueza escondida de las naciones: cómo funcionan los paraísos fiscales y qué hacer con ellos. México DF, México: Siglo Xxi.

Zucman, G. (2016, abril 20). Los paraísos fiscales exacerban la injusticia. Informático.com. Recuperado de http://informa-tico.com/20-04-2016/paraisos-fiscales-exacerban-injusticia 